\title{
NEUROMUSCULAR DISEASES CAUSING FLOPPY INFANT SYNDROME
}

\author{
V.Straub \\ Institute of Genetic Medicine, Newcastle University, Newcastle upon Tyne, UK
}

We refer to a floppy infant as an infant with generalized hypotonia presenting at birth or in early life. There is a broad spectrum of underlying conditions, which can either be acquired or of genetic origin, leading to a reduction in muscle tone and floppy infant syndrome. One aim when assessing a hypotonic infant is to localize the underlying cause of the condition to the upper or lower motor unit. The presence of hypotonia in combination with weakness in the limb muscles and reduced reflexes suggests a lesion of the lower motor unit or neuromuscular disease. The lesion can either be in the anterior horn cell, as in spinal muscular atrophy, the peripheral nerve, as in the hereditary neuropathies, the neuromuscular junction or the muscle fibre itself. Studies have suggested that about a third of floppy infants admitted to the neonatal intensive care unit are affected by a neuromuscular disease. In most cases the primary defect is within the muscle fibre resulting in a form of congenital myopathy, congenital muscular dystrophy or a metabolic myopathy. Diseases of the neuromuscular junction presenting as floppy infant syndrome can be acquired or hereditary. Over the recent years a growing number of congenital myasthenic syndromes has been identified, many of which can be treated if adequately diagnosed. With new therapies hovering above the horizon and in order to counsel families properly it becomes increasingly important to make a precise diagnosis and to understand the natural history of neuromuscular diseases causing floppy infant syndrome. 